\title{
Determinantal formulae for the Casimir operators of inhomogeneous Lie algebras
}

\author{
Rutwig Campoamor-Stursberg $\dagger$ \\ † Dpto. Geometría y Topología \\ Fac. CC. Matemáticas \\ Universidad Complutense de Madrid \\ Plaza de Ciencias, 3 \\ E-28040 Madrid, Spain \\ E-mail: rutwig@mat.ucm.es
}

\begin{abstract}
Contractions of Lie algebras are combined with the classical matrix method of Gel'fand to obtain matrix formulae for the Casimir operators of inhomogeneous Lie algebras. The method is presented for the inhomogeneous pseudounitary Lie algebras $I \mathfrak{u}(p, q)$. This procedure is extended to contractions of $I \mathfrak{u}(p, q)$ isomorphic to an extension by a derivation of the inhomogeneous special pseudo-unitary Lie algebras $I \mathfrak{s u}(p-1, q)$, providing an additional analytical method to obtain their invariants. Further, matrix formulae for the invariants of other inhomogeneous Lie algebras are presented.
\end{abstract}

PACS numbers: $02.20 \mathrm{~Sv}$ 


\section{Introduction}

Unitary Lie algebras constitute one of the most important classes of Lie algebras appearing in physics, as shows their application in hadron physics, the quark model or spontaneously broken symmetries. In combination with other Lie algebras, pseudounitary algebras are also relevant for physical phenomena, like the quaplectic group in the Born reciprocity. Generalized Inönü-Wigner contractions of these algebras are therefore also of interest, because they allow to relate certain model transitions, as happens for the quantum mechanical versions of the liquid drop model [1], where models exist whose dynamical group is respectively the unitary group $U(6)$ and the inhomogeneous unitary group $I U(5)$. Since the latter can be obtained by means of Inönü-Wigner contractions of $U(6)$, the corresponding models are related by a limiting process. Inhomogeneous unitary algebras have also been used to describe quantum states in relativistic theories of internal particle properties [2]. In all these problems, effective expressions for the Casimir operators of the unitary and inhomogeneous unitary algebras are needed. In contrast to the simple or reductive algebras, the computation of the invariants of inhomogeneous algebras $\mathfrak{g}$ is not feasible using the classical theory, and various approaches have been developed [3, 4, 5, 6], using either the universal enveloping algebra $\mathfrak{U}(\mathfrak{g})$ explicitly or by means of analytical reductions of differential equations.

In this work we analyze the generalization of the matrix procedure of Gel'fand [7] to various inhomogeneous Lie algebras. This generalization does not involve computations in the enveloping algebras or reductions of differential equations, but focuses on the combination of the Gel'fand method for simple Lie algebras with contractions of Lie algebras [8, 9]. We first compute the Casimir operators of inhomogeneous pseudounitary algebras $I \mathfrak{u}(p, q)$ by means of characteristic polynomials, and then extend the result to certain of its contractions. We also analyze the corresponding problem for inhomogeneous special pseudo-unitary algebras $I \mathfrak{s u}(p, q)$, which are obtained contracting $\ddagger$ either $\mathfrak{s u}(p+1, q)$ or $\mathfrak{s u}(p, q+1)$ [10]. However, here the contraction of the corresponding Casimir operators leads to dependence problems. To avoid this situation, we construct a special contraction $K \mathfrak{s u}(p, q)$ of $\mathfrak{s u}(p+1, q)$, which can be realized as an extension by a derivation of the inhomogeneous algebras $I \mathfrak{s u}(p, q)$. We give a determinantal formula for the invariants of this contraction, and determine a maximal set $\left\{W_{k}\right\}$ of invariants which satisfy a certain constraint. Analyzing how these functions $W_{k}$ transform when inserted in the system of PDEs giving the invariants of $I \mathfrak{s u}(p, q)$, we propose a simple analytical method to compute Casimir operators of the latter algebra. This will moreover explain why contraction of $\mathfrak{s u}(p, q)$-invariants leads to powers of the quadratic Casimir operator of $I \mathfrak{s u}(p, q)$, as already observed in [10]. We finally present matrix formulae to determine the Casimir invariants of other Lie algebras, namely the inhomogeneous Lie algebras $I \mathfrak{s o}(p, q)$ and two of its contractions that can be seen as a generalization of the classical Galilei and Carroll Lie algebras.

$\ddagger$ More specifically, as a direct summand of a contraction. 
The method to obtain the invariants of a Lie algebra that we will use in this work is the analytical approach, i.e., using the representation of $\mathfrak{g}$ by differential operators

$$
\widehat{X}_{i}=-C_{i j}^{k} x_{k} \frac{\partial}{\partial x_{j}}, \quad 1 \leq i \leq n,
$$

where $\left\{C_{i j}^{k}\right\}$ is the structure tensor over the basis $\left\{X_{1}, . ., X_{n}\right\}$ and $\left\{x_{1}, . ., x_{n}\right\}$ the dual basis. A function $F\left(x_{1}, . ., x_{n}\right)$ is an invariant if it satisfies the system:

$$
\widehat{X}_{i} F\left(x_{1}, . ., x_{n}\right)=-C_{i j}^{k} x_{k} \frac{\partial}{\partial x_{j}} F\left(x_{1}, . ., x_{n}\right)=0, \quad 1 \leq i \leq n
$$

If $F$ is a polynomial solution of (2), its symmetrization corresponds to a classical Casimir operator (see e.g. [3]). The number $\mathcal{N}(\mathfrak{g})$ of independent solutions of the system (non necessarily polynomials) is given by [1]:

$$
\mathcal{N}(\mathfrak{g})=\operatorname{dim} \mathfrak{g}-\operatorname{rank}\left(C_{i j}^{k} x_{k}\right),
$$

where $A(\mathfrak{g}):=\left(C_{i j}^{k} x_{k}\right)$ is the matrix which represents the commutator table over the basis $\left\{X_{1}, . ., X_{n}\right\}$.

We also recall the elementary notions about contractions that will be needed here. Let $\mathfrak{g}$ be a Lie algebra and $\Phi_{t} \in A u t(\mathfrak{g})$ a family of automorphisms of $\mathfrak{g}$, where $t \in \mathbb{N}$. For any $X, Y \in \mathfrak{g}$ define

$$
[X, Y]_{\Phi_{t}}:=\left[\Phi_{t}(X), \Phi_{t}(Y)\right]=\Phi_{t}([X, Y]) .
$$

Obviously $[X, Y]_{\Phi_{t}}$ are the brackets of the Lie algebra over the transformed basis. Now suppose that the limit

$$
[X, Y]_{\infty}:=\lim _{t \rightarrow \infty} \Phi_{t}^{-1}\left[\Phi_{t}(X), \Phi_{t}(Y)\right]
$$

exists for any $X, Y \in \mathfrak{g}$. Equation (5) defines a Lie algebra $\mathfrak{g}^{\prime}$ called the contraction of $\mathfrak{g}$ (by $\Phi_{t}$ ). If $\mathfrak{g}$ and $\mathfrak{g}^{\prime}$ are nonisomorphic, then the contraction is called non-trivial. This procedure can be enlarged to contract Casimir invariants. If $F\left(X_{1}, . ., X_{n}\right)$ is a Casimir operator of degree $p$ of $\mathfrak{g}$, we can rewrite it in the new basis $\left\{\Phi_{t}\left(X_{1}\right), . ., \Phi_{t}\left(X_{n}\right)\right\}$ and consider the limit

$$
F^{\prime}\left(X_{1}, . ., X_{n}\right):=\lim _{t \rightarrow \infty} t^{p} F\left(\Phi_{t}\left(X_{1}\right), . ., \Phi_{t}\left(X_{n}\right)\right) .
$$

It is straightforward to verify, using (51), that $F^{\prime}\left(X_{1}, . ., X_{n}\right)$ is a Casimir operator of the contraction. This method has become standard in the literature to obtain Casimir invariants of contractions [8, 9, 12, 13.

\section{Inhomogeneous pseudo-unitary Lie algebras and their invariants.}

In this work we will use the basis of $\mathfrak{u}(p, q)$ given by the operators $\left\{E_{\mu \nu}, F_{\mu \nu}\right\}_{1 \leq \mu, \nu \leq p+q=n}$ with the constraints $E_{\mu \nu}+E_{\nu \mu}=0, F_{\mu \nu}-F_{\nu \mu}=0$. The commutation relations over this basis are:

$$
\begin{aligned}
{\left[E_{\mu \nu}, E_{\lambda \sigma}\right] } & =g_{\mu \lambda} E_{\nu \sigma}+g_{\mu \sigma} E_{\lambda \nu}-g_{\nu \lambda} E_{\mu \sigma}-g_{\nu \sigma} E_{\lambda \mu} \\
{\left[E_{\mu \nu}, F_{\lambda \sigma}\right] } & =g_{\mu \lambda} F_{\nu \sigma}+g_{\mu \sigma} F_{\lambda \nu}-g_{\nu \lambda} F_{\mu \sigma}-g_{\nu \sigma} F_{\lambda \mu} \\
{\left[F_{\mu \nu}, F_{\lambda \sigma}\right] } & =g_{\mu \lambda} E_{\nu \sigma}+g_{\nu \lambda} E_{\mu \sigma}-g_{\nu \sigma} E_{\lambda \mu}-g_{\mu \sigma} E_{\lambda \nu}
\end{aligned}
$$


where $\left(g_{\mu \nu}\right)=(1, . ., 1,-1, . .,-1)$ is a diagonal matrix. Since $\mathfrak{u}(p, q)=\mathfrak{s u}(p, q) \oplus \mathbb{R}$, it follows at once that $\mathfrak{u}(p, q)$ has $(p+q)$ independent Casimir operators, one of them being $g^{\mu \mu} F_{\mu \mu}$, for corresponding to the central element, while the other $(p+q-1)$ invariants correspond to the simple part [14, 15, 16.

Proposition 1 For $N=p+q \geq 2$, a maximal set of independent Casimir invariants of $\mathfrak{u}(p, q)$ is given by the coefficients $C_{k}$ of the characteristic polynomial $\left|i A_{p, q}-\lambda \operatorname{Id}_{N}\right|=$ $\lambda^{N}+\sum_{k=1}^{N} C_{k} \lambda^{N-k}$, where

$$
A_{p, q}=\left(\begin{array}{ccccc}
-i f_{11} & . . & -g_{j j}\left(e_{1 j}+i f_{1 j}\right) & . . & -g_{N N}\left(e_{1 N}+i f_{1 N}\right) \\
: & & : & & : \\
e_{1 j}-i f_{1 j} & . . & -i g_{j j} f_{j j} & . . & -g_{N N}\left(e_{j N}+i f_{j N}\right) \\
: & & : & & : \\
e_{1 N}-i f_{1 N} & . . & g_{j j}\left(e_{j N}-i f_{j N}\right) & . . & -i g_{N N} f_{N N}
\end{array}\right)
$$

and $i=\sqrt{-1}$. Moreover, $\operatorname{deg}\left(C_{k}\right)=k$ for $k=1 . . p+q=N$.

In particular, if $g_{i i}=1$ for all $i$, we obtain the unitary algebra $\mathfrak{u}(n)$. Using the notation $a^{i j}=\left(e_{i j}+i f_{i j}\right)$ with the constraint $a^{i j}=-a^{j i *}$, it was shown in [18, chapter 7, p.239] that the Casimir operators of $\mathfrak{u}(n)$ are obtained from the secular equation

$$
\operatorname{det}\left\|a^{i j} J_{i j}-\lambda I_{n}\right\|=(-\lambda)^{n-r} \phi_{r}\left(a^{i j}\right) \text {, }
$$

where

$$
\phi_{r}\left(a^{i j}\right)=\frac{1}{r !(n-r) !} \varepsilon_{i_{1} . . i_{n}} a^{i_{1} j_{1}} \ldots a^{i_{r} j_{r}} \varepsilon_{j_{1} . ., j_{r}} .
$$

Here $J_{i j}$ is the fundamental matrix having entry 1 at position $(i, j)$ and zero elsewhere, and $\varepsilon$ the skew Levi-Civita tensor. Formula (10) is nothing but the corresponding adaptation to the different real forms of $\mathfrak{u}(N)$ over the basis (17)-(9).

There is an interesting consequence of this formulation, namely, that the determinant of $A_{p, q}$ satisfies the following identity

$$
\operatorname{det}\left(A_{p, q}\right)=\prod_{j=2}^{N} g_{j j} \operatorname{det}\left(A_{N, 0}\right) .
$$

This implies that all pseudo-unitary algebras $\mathfrak{u}(p, q)$ have the Casimir invariant of order $N$ in common, while those of lower degree depend essentially on the index and signature of the matrix $\left(g_{\mu \mu}\right)$.

As shown first by Rosen, inhomogeneous unitary Lie algebras $\operatorname{Iu}(p, q)$ can be obtained from an Inönü-Wigner contraction of the unitary algebras $\mathfrak{u}(p+1, q)$ or $\mathfrak{u}(p, q+1)$. We will use also this contraction to obtain the matrix formulae giving the Casimir operators of $I \mathfrak{u}(p, q)$, but without working explicitly with elements of the enveloping algebra. Our approach uses only matrices, and the invariants will follow from characteristic polynomials or combinations of them. To this extent, we must first fix some notation. We consider the ordered basis $\left\{F_{i i}, E_{i j}, F_{i j}\right\}$ for $1 \leq i, j \leq N$. Without 
loss of generality, we can suppose that for the matrix (10) we have $g_{11}=1$. We consider the automorphism $\Phi$ of $\mathfrak{u}(p, q)$ given by

$$
\begin{array}{llll}
\Phi\left(F_{11}\right)=\frac{1}{t} F_{11} ; & \Phi\left(F_{\mu \mu}\right)=F_{\mu \mu}(\mu \geq 2) ; & \Phi\left(E_{1 \sigma}\right)=\frac{1}{t} E_{1 \sigma}(\sigma \geq 2) \\
\Phi\left(F_{1 \sigma}\right)=\frac{1}{t} F_{1 \sigma}(\sigma \geq 2) & \Phi\left(E_{\mu \nu}\right)=E_{\mu \nu}, & \Phi\left(F_{\mu \nu}\right)=F_{\mu \nu}, \mu, \nu \neq 1 .
\end{array}
$$

For $\mu, \nu \neq 1$ the generators $\Phi\left(E_{\mu \nu}\right)$ and $\Phi\left(F_{\mu \nu}\right)$ obviously generate the unitary Lie algebra $\mathfrak{u}(p-1, q)$. The $\operatorname{limit}_{\lim _{t \rightarrow \infty}} \Phi^{-1}[\Phi(X), \Phi(Y)]$ exists for any pair of elements $X, Y \in \mathfrak{u}(p, q)$, and clearly defines a contraction. If we write $\Phi\left(F_{1 \rho}\right):=Q_{\rho}$ and $\Phi\left(E_{1 \rho}\right):=R_{\rho}$, then we have the brackets

$$
\begin{array}{ll}
{\left[E_{\mu \nu}, R_{\rho}\right]=g_{\mu \rho} R_{\nu}-g_{\nu \rho} R_{\mu},} & {\left[E_{\mu \nu}, Q_{\rho}\right]=g_{\mu \rho} Q_{\nu}-g_{\nu \rho} Q_{\mu}} \\
{\left[F_{\mu \nu}, Q_{\rho}\right]=-g_{\nu \rho} R_{\mu}-g_{\mu \rho} R_{\nu},} & {\left[F_{\mu \nu}, R_{\rho}\right]=g_{\mu \rho} Q_{\nu}+g_{\nu \rho} Q_{\mu}}
\end{array}
$$

and this coincides exactly with the standard representation of $\mathfrak{u}(p-1, q)$ as given in 10. Observe that in particular $\Phi\left(F_{11}\right)$ commutes with any other generator. Therefore the contraction is isomorphic to the direct sum $I \mathfrak{u}(p-1, q) \oplus \mathbb{R}$.

Let $N+1=p+q+1 \geq 2$ and consider the contraction $\mathfrak{u}(p, q) \rightsquigarrow I \mathfrak{u}(p-$ $1, q) \oplus\left\langle F_{11}\right\rangle$ determined by (14). We next give with an analogous formula for the inhomogeneous Lie algebra $I \mathfrak{u}(p-1, q)$.

Proposition 2 In the preceding conditions, a maximal set of independent Casimir operators $D_{k}$ of $I \mathfrak{u}(p-1, q)$ is obtained from the determinant $\Delta(\lambda)=\sum_{k=2}^{N} D_{k} \lambda^{N-k}$ defined by

$$
\lim _{t \rightarrow \infty} \frac{1}{t^{2}}\left|\begin{array}{ccccc}
f_{11} t-\lambda & \ldots & -g_{j j}\left(i e_{1 j}-f_{1 j}\right) t & \ldots & -g_{N+1, N+1}\left(i e_{1, N+1}-f_{1, N+1}\right) t \\
: & & : & & : \\
\left(i e_{1 j}+f_{1 j}\right) t & \ldots & g_{j j} f_{j j}-\lambda & \ldots & -g_{N+1, N+1}\left(i e_{j, N+1}-f_{j, N+1}\right) \\
: & & : & & : \\
\left(i e_{1, N+1}+f_{1, N+1}\right) t & \ldots & g_{j j}\left(i e_{j, N+1}+f_{j, N+1}\right) & \ldots & g_{N+1, N+1} f_{N+1, N+1}-\lambda
\end{array}\right|
$$

The preceding formula is obtained starting from (10) and implementing the contraction to the algebra $I \mathfrak{u}(p, q) \oplus \mathbb{R}$, scaling the determinant to avoid divergencies (see equation (6) ). The independence of the contracted invariants is proved directly. It follows from equation (10) that for any $2 \leq j \leq N$ following identities hold:

$$
\frac{\partial^{3} C_{j}}{\partial e_{1 \mu} \partial e_{1 \nu} \partial e_{1 \lambda}}=\frac{\partial^{3} C_{j}}{\partial e_{1 \mu} \partial e_{1 \nu} \partial f_{1 \lambda}}=\frac{\partial^{3} C_{j}}{\partial f_{1 \mu} \partial f_{1 \nu} \partial e_{1 \lambda}}=\frac{\partial^{3} C_{j}}{\partial f_{1 \mu} \partial f_{1 \nu} \partial f_{1 \lambda}}=0 .
$$

That means that any invariant $\oint$ of $\mathfrak{u}(p, q)$ can be written as a quadratic polynomial in the variables $\left\{e_{1 \mu}, f_{1 \mu}\right\}$ with coefficients in the polynomial ring $\mathbb{R}\left[e_{\mu \nu}, f_{\mu \nu}\right]_{2 \leq \mu, \nu \leq N}$. Moreover, it is straightforward to verify that no function depending only on the variables $\left\{e_{\mu \nu}, f_{\mu \nu}\right\}$ with $\mu, \nu \neq 1$ is an invariant of $I \mathfrak{u}(p, q)$, because of the the action of the reductive part $\mathfrak{u}(p-1, q)$ over the representation given in (15). From these facts it follows that the functions $D_{k}$ are algebraically independent homogeneous polynomials, and therefore their symmetrization are independent Casimir invariants [17. By algebraic $\S$ Of degree $d \geq 2$. 
manipulation, we can reduce and simplify the determinant (16) in order to obtain an expression that does not involve anymore the contraction parameter $t$. This is done applying the usual determinantal methods, like the Laplace expansion. After some decompositions and computations, we obtain that

$$
\Delta=\left|B_{p, q}-\lambda \operatorname{Id}_{N}\right|+\lambda\left|\left(B_{p, q}\right)_{11}-\lambda \operatorname{Id}_{N-1}\right|,
$$

where

$$
B_{p, q}:=\left(\begin{array}{ccccc}
0 & . . & g_{j j}\left(f_{1 j}-i e_{1 j}\right) & . . & g_{N+1, N+1}\left(f_{1, N+1}-i e_{1, N+1}\right) \\
: & & : & & : \\
\left(i e_{1 j}+f_{1 j}\right) & . . & g_{j j} f_{j j} & . . & g_{N+1, N+1}\left(f_{j, N+1}-i e_{j, N+1}\right) \\
: & & : & & : \\
\left(i e_{1, N+1}+f_{1, N+1}\right) & . . & g_{j j}\left(i e_{j, N+1}+f_{j, N+1}\right) & . . & g_{N+1, N+1} f_{N+1, N+1}
\end{array}\right),
$$

and $\left(B_{p, q}\right)_{11}$ is the minor obtained deleting the first row and column. Now, replacing $e_{1 j}$ by $r_{j}$ and $f_{1 j}$ by $q_{j}$, equation (19) gives the Casimir operators of $I \mathfrak{u}(p-1, q)$ over the usual basis $\left\{E_{\mu \nu}, F_{\mu \nu}, Q_{\rho}, R_{\rho}\right\}$. The advantage of formula (19) with respect to equation (16) is that the invariants are expressed in the usual basis of $I \mathfrak{u}(p, q)$, without taking into account that this algebra arises as contraction of the unitary algebra $\mathfrak{u}(p+1, q)$, and therefore avoiding the computation of limits.

\section{A comment concerning the special pseudo-unitary algebras}

Formula (10) can be easily adapted to the special algebras. For convenience we take the Cartan subalgebra spanned by the vectors $H_{\mu}=g_{\mu+1, \mu+1} F_{\mu \mu}-g_{\mu \mu} F_{\mu+1, \mu+1}$ for $\mu=1 . . p+q-1$. The centre of $\mathfrak{u}(p, q)$ is obviously generated by $g^{\mu \mu} F_{\mu \mu}$, which coincides with the first order invariant obtained previously.

Proposition 3 For $N \geq 2$, a maximal set of independent Casimir invariants of $\mathfrak{s u}(p, q)$ (where $p+q=N$ ) is given by the coefficients $D_{k}$ of the characteristic polynomial $\left|i A_{p, q}-\lambda \operatorname{Id}_{N}\right|=\lambda^{N}+\sum_{k=2}^{N} D_{k} \lambda^{N-k}$, where

$A_{p, q}=\left(\begin{array}{ccccc}-i Y_{1} & . . & -g_{\mu \mu}\left(e_{1 \mu}+i f_{1 \mu}\right) & . . & -g_{N N}\left(e_{1 N}+i f_{1 N}\right) \\ : & & : & & : \\ e_{1 \mu}-i f_{1 \mu} & . . & -i Y_{\mu} & . . & -g_{N N}\left(e_{\mu N}+i f_{\mu N}\right) \\ : & & : & & : \\ e_{1 N}-i f_{1 N} & . . & g_{\mu \mu}\left(e_{\mu N}-i f_{\mu N}\right) & . . & -i Y_{N}\end{array}\right)$

and

$$
Y_{\mu}=\sum_{\nu=1}^{\mu-1} \frac{-\nu}{N} g_{\nu \nu} g_{\nu+1, \nu+1} h_{\nu}+\sum_{\nu=\mu}^{N-1} \frac{N-\nu}{N} g_{\nu \nu} g_{\nu+1, \nu+1} h_{\nu}, 1 \leq \mu \leq N .
$$

Moreover, $\operatorname{deg}\left(D_{k}\right)=k$ for $k=2 . . p+q=N$. 
The interest of the preceding formula (20) is its application to obtain closed expressions for the Casimir operators of some contractions of $\mathfrak{s u}(p, q)$. Consider for instance the automorphism

$$
\begin{array}{lll}
\Phi\left(H_{\mu}\right)=H_{\mu}(\mu \geq 1), & \Phi\left(E_{1 \sigma}\right)=\frac{1}{t} E_{1 \sigma}(\sigma \geq 2), & \\
\Phi\left(F_{1 \sigma}\right)=\frac{1}{t} F_{1 \sigma}(\sigma \geq 2), & \Phi\left(E_{\mu \nu}\right)=E_{\mu \nu}, & \Phi\left(F_{\mu \nu}\right)=F_{\mu \nu}, \mu, \nu \neq 1 .
\end{array}
$$

For any pair of elements $X, Y$ of the algebra $\lim _{t \rightarrow \infty} \Phi^{-1}[\Phi(X), \Phi(Y)]$ exists, and defines a Lie algebra which will be denoted by $K \mathfrak{s u}(p-1, q)$.\| It follows at once from (22) that $K \mathfrak{s u}(p-1, q)$ is isomorphic to an extension of degree one of the inhomogeneous algebra $I \mathfrak{s u}(p-1, q)$. In fact, observe that the action of the Cartan subalgebra $\mathfrak{H}$ over the $E_{\mu, \nu}$ and $F_{\mu, \nu}$ is not changed by the contraction. If we consider the element $H^{\prime} \in \mathfrak{H}$ defined by

$$
H^{\prime}=\sum_{\mu=1}^{p+q-1}(p+q-\mu) \prod_{\rho \neq \mu, \mu+1} g_{\rho \rho} H_{\mu},
$$

it is not difficult to see that taking the new basis of $\mathfrak{H}$ generated by $\left\{H^{\prime}, H_{2}, . ., H_{p+q-1}\right\}$ we have $\left[H^{\prime}, E_{\mu \nu}\right]=\left[H^{\prime}, F_{\mu \nu}\right]=0$ and

$$
\left[H^{\prime}, E_{1 \mu}\right]=-2(p+q) \prod_{\rho=1}^{p+q} g_{\rho \rho} F_{1 \mu}, \quad\left[H^{\prime}, F_{1 \mu}\right]=2(p+q) \prod_{\rho=1}^{p+q} g_{\rho \rho} E_{1 \mu}
$$

for $2 \leq \mu<\nu$. This shows that $\left[H^{\prime}, \mathfrak{s u}(p-1, q)\right]=0$. Using this fact, it is straightforward to verify that the linear mapping $\varphi: I \mathfrak{s u}(p-1, q) \longrightarrow I \mathfrak{s u}(p-1, q)$ defined by $\varphi(X)=\left[H^{\prime}, X\right]$ is a derivation of the inhomogeneous algebra $I \mathfrak{s u}(p-1, q)$. This result allows us to determine the Casimir operators of the extension using the preceding formuale. The proof is completely analogous to that of proposition 2 .

Proposition 4 For $N=p+q \geq 2$, a maximal set of independent Casimir invariants of $K \mathfrak{s u}(p-1, q)$ over the basis $\left\{H^{\prime}, H_{2}, . ., H_{N-1}, E_{\mu \nu}, F_{\mu \nu}\right\}$ is given by the coefficients $W_{k}$ of the polynomial

$$
\left|i C_{p, q}-\lambda \operatorname{Id}_{N}\right|+\lambda\left|i\left(C_{p, q}\right)_{11}-\lambda \operatorname{Id}_{N-1}\right|=\sum_{k=2}^{N} W_{k} \lambda^{N-k}
$$

where

$$
C_{p, q}=\left(\begin{array}{ccccc}
0 & . . & -g_{\mu \mu}\left(e_{1 \mu}+i f_{1 \mu}\right) & . . & -g_{N N}\left(e_{1 N}+i f_{1 N}\right) \\
: & & : & & : \\
e_{1 \mu}-i f_{1 \mu} & . . & -i Y_{\mu} & . . & -g_{N N}\left(e_{\mu N}+i f_{\mu N}\right) \\
: & & : & & : \\
e_{1 N}-i f_{1 N} & . . & g_{\mu \mu}\left(e_{\mu N}-i f_{\mu N}\right) & . . & -i Y_{N}
\end{array}\right) \text {, }
$$

and

$Y_{\mu}=-\prod_{\rho=1}^{N} g_{\rho \rho} \frac{h^{\prime}}{N(N-1)}+\sum_{\nu=2}^{\mu-1} \frac{1-\nu}{N-1} g_{\nu \nu} g_{\nu+1, \nu+1} h_{\nu}+\sum_{\nu=\mu}^{N-1} \frac{N-\nu}{N-1} g_{\nu \nu} g_{\nu+1, \nu+1} h_{\nu}$

for $2 \leq \mu \leq N$. Moreover, $\operatorname{deg}\left(W_{k}\right)=k$ for $k=2 . . p+q=N$.

$\|$ The Levi part, which is isomorphic to $\mathfrak{s u}(p-1, q)$, is generated by $\left\{H_{\mu}, E_{\mu \nu}, F_{\mu \nu}\right\}_{2 \leq \mu<\nu}$. 
The functions $W_{k}$ have a structural property that will be of interest in the analysis of the Casimir operators of inhomogeneous algebra $I \mathfrak{s u}(p, q)$.

Proposition 5 For any $N \geq 2$, the functions $W_{k}$ of (25) satisfy the equation

$$
N(N-1) \frac{\partial W_{k}}{\partial h^{\prime}}-(N+1-k) \prod_{\rho=1}^{N} g_{\rho \rho} W_{k-1}=0 .
$$

Proof. By (25), the $W_{k}$ are the result of a combination of the characteristic polynomial of the matrix $i C_{p, q}$ and the minor $i\left(C_{p, q}\right)$ obtained deleting the first row and column. Therefore, using the expansion properties of determinants, it follows easily that any $W_{k}$ is obtained from the sum of the minors of order $k$ of $i C_{p, q}$ that contain the first column and row, i.e.,

$$
W_{k}=\sum_{2<\mu_{1} . .<\mu_{k-1}} M\left(1, \mu_{1}, . ., \mu_{k-1}\right)
$$

where $M\left(1, \mu_{1}, . ., \mu_{k-1}\right)$ is the matrix given by:

$$
\left(\begin{array}{cccc}
0 & -g_{\mu_{1} \mu_{1}}\left(i e_{1 \mu_{1}}-f_{1 \mu_{1}}\right) & . . & -g_{\mu_{k-1}, \mu_{k-1}}\left(i e_{1 \mu_{k-1}}-f_{1 \mu_{k-1}}\right) \\
\left(i e_{1 \mu_{1}}+f_{1 \mu_{1}}\right) & Y_{\mu_{1}} & . . & -g_{\mu_{k-1}, \mu_{k-1}}\left(i e_{\mu_{1} \mu_{k-1}}-f_{\mu_{1} \mu_{k-1}}\right) \\
: & : & & : \\
\left(i e_{1 \mu_{k-1}}+f_{1 \mu_{k-1}}\right) & g_{\mu_{1} \mu_{1}}\left(i e_{\mu_{1} \mu_{k-1}}+f_{\mu_{1} \mu_{k-1}}\right) & . . & Y_{\mu_{k-1}}
\end{array}\right) .
$$

Now the variable $h^{\prime}$ is contained only in the elements $Y_{\mu}$ of the diagonal, which implies that

$$
\begin{aligned}
& \frac{\partial W_{k}}{\partial h^{\prime}}=-\sum_{2<\mu_{1 . .}<\mu_{k-1}} \frac{\partial M\left(1, \mu_{1}, . ., \mu_{k-1}\right)}{\partial h^{\prime}}=-\sum_{2<\mu_{1} . .<\mu_{k-1}} \sum_{j=\mu_{1}}^{\mu_{k-1}} \frac{\partial Y_{j}}{\partial h^{\prime}} M\left(1, \mu_{1}, . ., \mu_{k-2}\right)= \\
& =\frac{(N+1-k)}{N(N-1)} \prod_{\rho=1}^{N} g_{\rho \rho} \sum_{2<\mu_{1} . .<\mu_{k-2}} M\left(1, \mu_{1}, . ., \mu_{k-2}\right)=\frac{(N+1-k)}{N(N-1)} \prod_{\rho=1}^{N} g_{\rho \rho} W_{k-1} .
\end{aligned}
$$

Since $K \mathfrak{s u}(p-1, q)$ is a contraction of $\mathfrak{s u}(p, q)$, by transitivity of contractions it follows that $K \mathfrak{s u}(p-1, q)$ also contracts onto $I \mathfrak{s u}(p-1, q) \oplus<H^{\prime}>$, and we obtain the chain of contractions

$$
\mathfrak{s u}(p, q) \rightsquigarrow K \mathfrak{s u}(p-1, q) \rightsquigarrow I \mathfrak{s u}(p-1, q) \oplus<H^{\prime}>.
$$

The question is whether the procedure developed to compute the Casimir invariants can be adapted to the special inhomogeneous case. The following example shows that in general, the contraction of the invariants forming a fundamental set of invariants of $\mathfrak{s u}(p, q)$ (or $K \mathfrak{s u}(p-1, q))$ does not generate a complete set of invariants of the contraction. Consider the Lie algebra $\mathfrak{s u}(3,1)$. This algebra has three invariants $C_{2}, C_{3}, C_{4}$ which are obtained from the characteristic polynomial of the matrix

$$
D=\left(\begin{array}{cccc}
\frac{3}{4} h_{1}+\frac{1}{2} h_{2}-\frac{1}{4} h_{3} & -i e_{12}+f_{12} & -i e_{13}+f_{13} & i e_{14}-f_{14} \\
i e_{12}+f_{12} & -\frac{1}{4} h_{1}+\frac{1}{2} h_{2}-\frac{1}{4} h_{3} & -i e_{23}+f_{23} & i e_{24}-f_{24} \\
i e_{13}+f_{13} & i e_{23}+f_{23} & -\frac{1}{4} h_{1}-\frac{1}{2} h_{2}-\frac{1}{4} h_{3} & i e_{34}-f_{34} \\
i e_{14}+f_{14} & i e_{24}+f_{24} & i e_{34}+f_{34} & -\frac{1}{4} h_{1}-\frac{1}{2} h_{2}+\frac{3}{4} h_{3}
\end{array}\right)
$$


We obtain the inhomogeneous algebra $I \mathfrak{s u}(2,1)$ from the contraction determined by the automorphism

$$
\begin{array}{lll}
\Phi\left(H_{1}\right)=\frac{1}{t} H_{1}, & \Phi\left(F_{\mu}\right)=F_{\mu}(\mu \geq 2), & \Phi\left(E_{1 \sigma}\right)=\frac{1}{t} E_{1 \sigma}(\sigma \geq 2), \\
\Phi\left(F_{1 \sigma}\right)=\frac{1}{t} F_{1 \sigma}(\sigma \geq 2), & \Phi\left(E_{\mu \nu}\right)=E_{\mu \nu}, & \Phi\left(F_{\mu \nu}\right)=F_{\mu \nu}, \mu, \nu \neq 1 .
\end{array}
$$

However, since we are rescaling also a generator of the Cartan subalgebra, it will follow that the contraction of the Casimir operators $C_{i}\left(h_{\mu}, e_{\mu \nu}, f_{\mu \nu}\right)$ for $i=2,3,4$ are functions of the quadratic operator $I_{2}=g_{\mu \mu}\left(e_{1 \mu}^{2}+f_{1 \mu}^{2}\right)$ of $I \mathfrak{s u}(2,1)$ and $h_{1} \boldsymbol{\Upsilon}$. More specifically, for $k=2,3,4$ we have:

$$
\lim _{t \rightarrow \infty} \frac{1}{t^{k}} C_{k}=-\alpha_{k} I_{2} h_{1}^{k-2}
$$

where $\alpha_{2}=1, \alpha_{3}=\frac{1}{2}, \alpha_{4}=\frac{3}{16}$. Therefore the characteristic polynomial of the matrix (33) does not provide a maximal set of invariants of $I \mathfrak{s u}(2,1)$. However, it is easy to obtain an independent invariant using (35). This third invariant, which must depend on all variables of $I \mathfrak{s u}(2,1)$ [6], can be obtained for example contracting the invariant $C_{2} C_{4}-\frac{1}{4} C_{3}^{2}$, and provides a sixth order Casimir operator of $I \mathfrak{s u}(2,1)$ which is not a power of $I_{2}$. A similar result holds if we start from the invariants of $K \mathfrak{s u}(2,1)$. We can however proceed differently. Instead of contracting the invariants $W_{k}$, we evaluate them in the system (2) corresponding to $I \mathfrak{s u}(2,1)$ over the basis $\left\{H_{2}, H_{3}, E_{\mu \nu}, F_{\mu \nu}\right\}$. We obtain

$$
\begin{array}{lll}
\widehat{H}_{\mu}\left(W_{i}\right)=0(\mu=2,3), & \widehat{E}_{\mu \nu}\left(W_{2}\right)=0, & \widehat{F}_{\mu \nu}\left(W_{2}\right)=0 \\
\widehat{E}_{\mu \nu}\left(W_{3}\right)=-\frac{4}{3} f_{\mu \nu} W_{2}, & \widehat{F}_{\mu \nu}\left(W_{3}\right)=\frac{4}{3} e_{\mu \nu} W_{2}, & \widehat{E}_{\mu \nu}\left(W_{4}\right)=-\frac{2}{3} f_{\mu \nu} W_{3} .
\end{array}
$$

Now let $u=W_{3}$ and $v=W_{4}$. For any $X \in\left\{E_{\mu \nu}, F_{\mu \nu}\right\}$ we have $\frac{\widehat{X}(v)}{\widehat{X}(u)}=\frac{W_{3}}{2 W_{2}}=\frac{u}{2 W_{2}}$. If we now consider the differential equation

$$
\frac{\partial F}{\partial u}+\frac{u}{2 W_{2}} \frac{\partial F}{\partial v}=0
$$

it follows easily that the solutions are generated by the function $\left(W_{2} v-\frac{1}{4} u^{2}\right) W_{2}^{-1}=$ $\left(W_{2} W_{4}-\frac{1}{4} W_{3}^{2}\right) W_{2}^{-1}$. Since $W_{2}$ is already an invariant of $I \mathfrak{s u}(2,1)$, this implies that $I=4 W_{2} W_{4}-W_{3}^{2}$ is an invariant of the algebra of degree six. A short computation shows that it coincides with $\lim _{t \rightarrow \infty} \frac{1}{t^{4}}\left(C_{2} C_{4}-\frac{1}{4} C_{3}^{2}\right)$. This fact is not casual and can be formulated in any dimension.

Theorem 1 Let $p+q=N \geq 2$ and $W_{k}$ be the invariants of the Lie algebra $K \mathfrak{s u}(p-1, q)$. A function $G\left(W_{2}, . ., W_{N}\right)$ is an invariant of the inhomogeneous Lie algebra $I \mathfrak{s u}(p-1, q)$ if and only if

$$
\frac{\partial G\left(W_{2}, . ., W_{N}\right)}{\partial h^{\prime}}=0
$$

Proof. Let us consider the bases of $K \mathfrak{s u}(p-1, q)$ and $I \mathfrak{s u}(p-1, q)$ used previously,and let $\left\{\widehat{H}^{\prime}, \widehat{H}_{\mu}, \widehat{E}_{\mu \nu}, \widehat{F}_{\mu \nu}\right\}$ and $\left\{\widetilde{H}^{\prime}, \widetilde{H}_{\mu}, \widetilde{E}_{\mu \nu}, \widetilde{F}_{\mu \nu}\right\}$ be the representation by I Observe that in the contraction this becomes a central element. 
differential operators of $K \mathfrak{s u}(p-1, q)$, respectively $I \mathfrak{s u}(p-1, q) \oplus<H^{\prime}>$. Since $K \mathfrak{s u}(p-1, q)$ is an extension by a derivation of $I \mathfrak{s u}(p-1, q)$ acting trivially on the Levi part, for the invariants $W_{k}$ we obtain the relations

$$
\begin{aligned}
& \widehat{H}_{\mu}\left(W_{\lambda}\right)=\widetilde{H}_{\mu}\left(W_{\lambda}\right)=0,1 \leq \mu \leq N-1 \\
& \widehat{E}_{\mu \nu}\left(W_{\lambda}\right)=\widetilde{E}_{\mu \nu}\left(W_{\lambda}\right)=0,2 \leq \mu, v \leq N \\
& \widehat{F}_{\mu \nu}\left(W_{\lambda}\right)=\widetilde{F}_{\mu \nu}\left(W_{\lambda}\right)=0,2 \leq \mu, v \leq N \\
& \widehat{E}_{1 \mu}\left(W_{\lambda}\right)=\widetilde{E}_{1 \mu}\left(W_{\lambda}\right)+2 N \prod_{\rho=1}^{N} g_{\rho \rho} \frac{\partial W_{\lambda}}{\partial h^{\prime}} f_{1 \mu}=0,2 \leq \mu \leq N \\
& \widehat{F}_{1 \mu}\left(W_{\lambda}\right)=\widetilde{F}_{1 \mu}\left(W_{\lambda}\right)-2 N \prod_{\rho=1}^{N} g_{\rho \rho} \frac{\partial W_{\lambda}}{\partial h^{\prime}} e_{1 \mu}=0,2 \leq \mu \leq N .
\end{aligned}
$$

For $W_{2}$ we obviously have $\frac{\partial W_{2}}{\partial h^{\prime}}=0$, we thus recover the quadratic invariant. For any $\lambda \geq 3$ the previous system, combined with proposition 5 , provides the following expressions for any $2 \leq \mu \leq N$ :

$$
\begin{aligned}
& \widetilde{E}_{1 \mu}\left(W_{\lambda}\right)=-2 N \prod_{\rho=1}^{N} g_{\rho \rho} \frac{\partial W_{\lambda}}{\partial h^{\prime}}=-\frac{2(N+1-k)}{(N-1)} W_{\lambda-1} f_{1 \mu}, \\
& \widetilde{F}_{1 \mu}\left(W_{\lambda}\right)=2 N \prod_{\rho=1}^{N} g_{\rho \rho} \frac{\partial W_{\lambda}}{\partial h^{\prime}}=\frac{2(N+1-k)}{(N-1)} W_{\lambda-1} e_{1 \mu} .
\end{aligned}
$$

As a consequence of these identities, an invariant $W$ of $K \mathfrak{s u}(p-1, q)$ is also an invariant of the inhomogeneous algebra $I \mathfrak{s u}(p-1, q)$ if and only if $\frac{\partial W}{\partial h^{\prime}}=0$.

Observe that equations (43) and (44) can be used to compute additional invariants of $I \mathfrak{s u}(p-1, q)$ using differential equations of the type (37). The fact that the resulting function must be independent of $h^{\prime}$ will impose some restrictions on the degree.

\section{On the applicability of the method}

Although the method to compute Casimir invariants by means of characteristic polynomials has been developed for the Lie algebras $\mathfrak{u}(p, q)$ and their contractions, the argument is not exclusive of the unitary case, and does not depend on the special shape of their invariants. In this section we give matrix formulae for the invariants of other inhomogeneous Lie algebras of physical importance. More specifically, we apply the method to the inhomogeneous pseudo-orthogonal Lie algebras, a double inhomogeneous algebra and a semidirect product of a Heisenberg and an orthogonal Lie algebra, which can both be obtained from contractions of $I \mathfrak{s o}(p, q)$ and were studied in [4].

\subsection{Inhomogeneous pseudo-orthogonal Lie algebras}

The inhomogeneous pseudo-orthogonal algebra $I \mathfrak{s o}(p, q)$ with $N=p+q$ is given by the $\frac{1}{2} N(N+1)$ operators $E_{\mu \nu}=-E_{\nu \mu}, P_{\mu}$, satisfying:

$$
\left[E_{\mu \nu}, E_{\lambda \sigma}\right]=g_{\mu \lambda} E_{\nu \sigma}+g_{\mu \sigma} E_{\lambda \nu}-g_{\nu \lambda} E_{\mu \sigma}-g_{\nu \sigma} E_{\lambda \mu}
$$




$$
\left[E_{\mu \nu}, P_{\rho}\right]=g_{\mu \rho} P_{\nu}-g_{\nu \rho} P_{\mu},
$$

where $g=\operatorname{diag}(1, . ., 1,-1, . .,-1)$. Moreover, in [3] it was shown that $\mathcal{N}(\operatorname{Iso}(p, q))=$ $\left[\frac{p+q+1}{2}\right]$.

Proposition 6 A maximal set of Casimir operators of $I \mathfrak{s o}(p, q)$ is given by the coefficients $C_{k}$ of $P(T)$ defined by

$$
P(T):=\left|B_{p, q}-T \operatorname{Id}_{N+1}\right|+T\left|\left(B_{p, q}\right)_{(N+1, N+1)}-T \operatorname{Id}_{N}\right|,
$$

where

$$
B_{p, q}:=\left(\begin{array}{cccccc}
0 & . . & -g_{j j} e_{1 j} & . . & -g_{N N} e_{1, N} & p_{1} T \\
: & & : & & : & \\
e_{1 j} & . . & 0 & . . & -g_{N N} e_{j, N} & p_{j} T \\
: & & : & & : & \\
e_{1, N} & . . & g_{j j} e_{j, N} & . . & 0 & p_{N} T \\
-p_{1} & & -g_{j j} p_{j} & & -g_{N N} p_{N} & 0
\end{array}\right)
$$

The proof is quite similar to that of proposition 2. Here we use the contraction of $\mathfrak{s o}(p+1, q)$ over $I \mathfrak{s o}(p, q)$, as done in [10]. In contrast to the unitary case, where the matrices involved are complex, here no problem of dependence in the contracted invariants is observed.

\subsection{The double inhomogeneous algebra $A G(p, q)$}

The Lie algebra $A G(p, q)$ with $N=p+q+1$ is given by the $\frac{1}{2}(N+1) N$ operators $E_{\mu \nu}=-E_{\nu \mu}, P_{\mu}, G_{\nu}, P_{0}$, satisfying the nontrivial commutation relations:

$$
\begin{array}{ll}
{\left[E_{\mu \nu}, E_{\lambda \sigma}\right]=g_{\mu \lambda} E_{\nu \sigma}+g_{\mu \sigma} E_{\lambda \nu}-g_{\nu \lambda} E_{\mu \sigma}-g_{\nu \sigma} E_{\lambda \mu},} & {\left[P_{0}, Q_{\rho}\right]=-g_{N-1, N-1} P_{\rho},} \\
{\left[E_{\mu \nu}, P_{\rho}\right]=g_{\mu \rho} P_{\nu}-g_{\nu \rho} P_{\mu},} & {\left[E_{\mu \nu}, Q_{\rho}\right]=g_{\mu \rho} Q_{\nu}-g_{\nu \rho} Q_{\mu},}
\end{array}
$$

where $1 \leq \mu, \nu, \rho, \leq N-1$ and $\left(g_{\mu \nu}\right)$ is the preceding diagonal matrix. It can be easily shown that this algebra is obtained by an Inönü-Wigner contraction of the inhomogeneous Lie algebra $I \mathfrak{s o}(p+1, q)$, considering the automorphism

$$
\begin{array}{ll}
\Psi\left(E_{\mu \nu}\right)=E_{\mu \nu}(1 \leq \mu, \nu \leq N-1), & \Psi\left(E_{\mu N}\right)=\frac{1}{t} E_{\mu N}:=G_{\mu} \\
\Psi\left(P_{\mu}\right)=\frac{1}{t} P_{\mu}(1 \leq \mu \leq N-1), & \Psi\left(P_{N}\right)=P_{N}:=P_{0} .
\end{array}
$$

This contraction preserves the number of invariants, i.e., $\mathcal{N}\left(A G(p, q)=\left[\frac{p+q+1}{2}\right]\right.$. Moreover, it should be remarked that $A G(p, q)$ is a double inhomogeneous Lie algebra with semidirect product structure

$$
A G(p, q)=I I \mathfrak{s o}(p, q)=\left(\mathfrak{s o}(p, q) \vec{\oplus} I_{N-1}\right) \vec{\oplus} I_{N},
$$

$I_{N}$ being the standard vector representation. In particular, for $q=0$ we obtain the proper Galilei algebra $I I \mathfrak{s o}(N-1)$. 
Proposition 7 A maximal set of Casimir operators of $A G(p, q)$ is given by the coefficients $C_{k}$ of the determinant $P(T)$ defined by

$$
P(T)=\left|\begin{array}{ccccccc}
-T & . . & -g_{\mu \mu} e_{1 \mu} & . . & -g_{N-1, N-1} e_{1, N-1} & -g_{N, N} q_{1} & p_{1} T \\
: & & : & & : & & \\
e_{1 \mu} & . . & -T & . . & -g_{N-1, N-1} e_{\mu, N-1} & -g_{N, N} q_{\mu} & p_{\mu} T \\
: & & : & & : & & \\
e_{1, N-1} & . . & g_{\mu \mu} e_{\mu, N-1} & . . & -T & -g_{N, N} q_{N-1} & p_{N-1} T \\
q_{1} & & g_{\mu \mu} q_{\mu} & & g_{N-1, N-1} q_{N-1} & 0 & 0 \\
-p_{1} & & -g_{\mu \mu} p_{\mu} & & -g_{N-1, N-1} p_{N-1} & 0 & 0
\end{array}\right|+
$$

\subsection{The Lie algebra $C(p, q)$}

The Lie algebra $C(p, q)$ with $N=p+q+1$ is also obtained from a contraction of $I \mathfrak{s o}(p+1, q)$ by means of the automorphism defined by

$$
\begin{array}{ll}
\Psi\left(E_{\mu \nu}\right)=E_{\mu \nu}(1 \leq \mu, \nu \leq N-1), & \Psi\left(E_{\mu N}\right)=\frac{1}{t} E_{\mu N}:=G_{\mu} \\
\Psi\left(P_{\mu}\right)=\frac{1}{t} P_{\mu}(1 \leq \mu \leq N-1), & \Psi\left(P_{N}\right)=\frac{1}{t^{2}} P_{N}:=P_{0} .
\end{array}
$$

Over the basis $\left\{E_{\mu \nu}, P_{\mu}, G_{\nu}, P_{0}\right\}$, the nontrivial commutation relations are:

$$
\begin{array}{ll}
{\left[E_{\mu \nu}, E_{\lambda \sigma}\right]=g_{\mu \lambda} E_{\nu \sigma}+g_{\mu \sigma} E_{\lambda \nu}-g_{\nu \lambda} E_{\mu \sigma}-g_{\nu \sigma} E_{\lambda \mu},} & {\left[P_{\rho}, Q_{\rho}\right]=-g_{\rho \rho} P_{0},} \\
{\left[E_{\mu \nu}, P_{\rho}\right]=g_{\mu \rho} P_{\nu}-g_{\nu \rho} P_{\mu},} & {\left[E_{\mu \nu}, Q_{\rho}\right]=g_{\mu \rho} Q_{\nu}-g_{\nu \rho} Q_{\mu},}
\end{array}
$$

where $1 \leq \mu, \nu, \rho, \leq N-1$. This algebra is isomorphic to the semidirect product of $\mathfrak{s o}(p, q)$ and the Heisenberg Lie algebra $\mathfrak{h}_{N}$, and therefore can be written as

$$
C(p, q)=I I^{\prime} \mathfrak{s o}(p, q)=\left(\mathfrak{s o}(p, q) \vec{\oplus} I_{N-1}^{\prime}\right) \vec{\oplus} I_{N},
$$

where the orthogonal algebra acts on $I_{N}$ through the vector representation and on $I_{N-1}^{\prime}$ through the contragredient of the vector representation. For $q=0$ we obtain the generalization of the proper Carroll algebra $C(p, 0)=I I^{\prime} \mathfrak{s o}(N-1)$. In particular it follows from (53) that $\mathcal{N}(C(p, q))=\left[\frac{p+q+1}{2}\right]$.

Proposition 8 A maximal set of Casimir operators of $C(p, q)$ is given by the coefficients $C_{k}$ of the determinant $P(T)$ defined by

$$
P(T)=\left|\begin{array}{ccccccc}
-T & . . & -g_{\mu \mu} e_{1 \mu} & . . & -g_{N-1, N-1} e_{1, N-1} & -g_{N, N} q_{1} & p_{1} T \\
: & & : & & : & & \\
e_{1 \mu} & . . & -T & . . & -g_{N-1, N-1} e_{\mu, N-1} & -g_{N, N} q_{\mu} & p_{\mu} T \\
: & & : & & : & & \\
e_{1, N-1} & . . & g_{\mu \mu} e_{\mu, N-1} & . . & -T & -g_{N, N} q_{N-1} & p_{N-1} T \\
q_{1} & & g_{\mu \mu} q_{\mu} & & g_{N-1, N-1} q_{N-1} & 0 & p_{0} T \\
-p_{1} & & -g_{\mu \mu} p_{\mu} & -g_{N-1, N-1} p_{N-1} & -g_{N, N} p_{0} & 0
\end{array}\right| .
$$




\section{Concluding remarks}

We have seen that, in analogy with the classical algebras, the Casimir operators of inhomogeneous pseudo-unitary Lie algebras $I \mathfrak{u}(p, q)$ can be computed using only characteristic polynomials, avoiding the use of the enveloping algebra 3. This formula is derived from the Inönü-Wigner contraction

$$
\mathfrak{u}(p, q) \rightsquigarrow I \mathfrak{u}(p-1, q) \oplus \mathbb{R},
$$

and allows to give a closed matrix expression for the invariants of the inhomogeneous algebras. The method can be enlarged to cover other contractions which are not necessarily inhomogeneous algebras. After adapting the formula to the special pseudounitary algebras $\mathfrak{s u}(p, q)$, we analyze the problem of computing the invariants for the inhomogeneous special algebras $I \mathfrak{s u}(p-1, q)$ applying the same procedure. However, the contraction of the invariants of $\mathfrak{s u}(p, q)$ points out that the contraction of independent Casimir invariants does not necessarily lead to independent Casimir operators of the contraction. This fact is the consequence of altering the Cartan subalgebra in the contraction. To solve this problem, special pseudo-unitary algebras are contracted onto an extension $K \mathfrak{s u}(p, q)$ of the inhomogeneous algebra $I \mathfrak{s u}(p, q)$. The invariants $W_{k}$ of this extension are shown to satisfy a functional equation (see (28) ) that can be used

to compute the invariants of $I \mathfrak{s u}(p, q)$ by purely analytical methods. This procedure enlarges the extension method introduced in $[13$ for the special affine algebras $\mathfrak{s a}(n, \mathbb{R})$ and other algebras having only one Casimir operator.

Further, we have exhibited examples that show the validity of the argument when applied to other Lie algebras different from unitary algebras. In particular, matrix formulae for the Casimir operators of the inhomogeneous pseudo-orthogonal Lie algebras and two Inönü-Wigner contractions that naturally generalize the generalized Galilei and Carroll algebras have been given. The latter contractions of $I \mathfrak{s o}(p+1, q)$ preserve the number of invariants [4]. Moreover, the structure of $C(p, q)$ shows that the procedure can be still valid for contractions of Lie algebras that are no more inhomogeneous.

\section{Acknowledgements}

The author expresses his gratitude to the referees for drawing the attention to some mistakes, as well as for valuable suggestions that helped to improve the manuscript. During the preparation of this work, the author was partially supported by the research project PR1/05-13283 of the U.C.M.

\section{References}

[1] Gilmore R, Feng D H, Vallieres M and Wood J L 1983 Phys. Rev. Letters C28 903

[2] Perjés Z 1979 Phys. Rev. D20 1857

[3] Demichev A P, Nelipa N F and Chai'chian M 1983 Comm. Math. Phys. 90353

[4] Herranz F J, Santander M 1997 J. Phys. A: Math. Gen. 305411 
[5] Pecina Cruz J N 1994 J. Math. Phys. 353146

[6] Campoamor-Stursberg R 2003 J. Phys. A: Math. Gen. 361357

[7] Gel'fand I M 1950 Mat. Sbornik 26112

[8] Herranz F J, Perez Bueno J C and Santander M 1998 J. Phys. A: Math. Gen. 315327

[9] Lykhmus Ya Kh 1969 Predel'nye (szhatye) gruppy Li Tartu

[10] Rosen J J. Math. Phys. 91305

[11] Beltrametti E G and Blasi A 1966 Phys. Lett. 2062

[12] Campoamor-Stursberg R 2003 Acta Phys. Polonica 34 3901; 352059.

[13] Campoamor-Stursberg R 2003 Phys. Lett. A 312211

[14] Racah G 1951 Group Theory and Spectroscopy (Princeton Univ. Press, N. J.)

[15] Perelomov A M and Popov V S 1967 Yad. Fiz 3924

[16] Campoamor-Stursberg R 2004 Phys. Lett. A $\mathbf{3 2 7} 138$

[17] Abellanas L and Martínez Alonso L 1975 J. Math. Phys. 161580

[18] Gilmore R 1974 Lie groups, Lie algebras and some of its applications (New York: Wiley) 